\title{
AN ASYMPTOTIC FORMULA IN THE THEORY OF NUMBERS
}

\author{
BY \\ H. HALBERSTAM
}

Introduction. Ramanujan $\left({ }^{1}\right)$ conjectured that if $\alpha$ and $\beta$ are fixed positive numbers

$$
\sum_{\nu=1}^{n-1} \sigma_{\alpha}(\nu) \sigma_{\beta}(n-\nu)-\frac{\Gamma(\alpha+1) \Gamma(\beta+1)}{\Gamma(\alpha+\beta+2)} \frac{\zeta(\alpha+1) \zeta(\beta+1)}{\zeta(\alpha+\beta+2)} \sigma_{\alpha+\beta+1}=o\left(\sigma_{\alpha+\beta+1}(n)\right)
$$

where $\sigma_{\alpha}(n)$ is the sum of the $\alpha$ th powers of the divisors of $n$.

In 1927 Ingham $\left({ }^{2}\right)$ published a similar result about the sum

$$
\sum_{v=1}^{n} d(\nu) d(n-\nu)
$$

where $d(n)$ is the number of divisors of $n$, and pointed out that his elementary method would serve also to confirm Ramanujan's conjecture. The proof of this was carried out in detail by the author of the present note $\left({ }^{3}\right)$, who proved, in addition, that if the left-hand side of Ramanujan's formula is denoted by $E(n)$,

$$
E(n)= \begin{cases}O\left(n^{\omega} \sigma_{-1}(n) \log n\right) & \text { when } \alpha=\beta<1 \\ O\left(n^{\omega} \log ^{c} n\right) & \text { otherwise; }\end{cases}
$$

here $\omega=\alpha+\beta+1-\min (\alpha, \beta, 1)$ and $c=0$ when $\min (\alpha, \beta)>1 ;$ when $\min (\alpha, \beta)$ $\leqq 1, c=0$ if the three numbers $\alpha, \beta, 1$ are different, $c=1$ if two of these numbers, but not all three, are equal, and $c=2$ if $\alpha=\beta=1$.

If $\min (\alpha, \beta) \geqq 1$, there is a saving of 1 in the exponent of the error term, and it appears difficult to improve this result. If, however, $\min (\alpha, \beta)<1$, and the added restriction

$$
\alpha+\beta<1
$$

is imposed, it is possible to adapt an analytic argument which Estermann( $\left.{ }^{4}\right)$ used to sharpen the above-mentioned result of Ingham, to proving the following:

Received by the editors April 12, 1956.

(1) Collected papers, p. 137.

(2) J. London Math. Soc. vol. 12 (1927) pp. 202-208.

(3) See Halberstam [1].

(4) See Estermann [2]. 
THEOREM.

$$
\begin{aligned}
\sum_{-1}^{n-1} \sigma_{\alpha}(\nu) \sigma_{\beta}(n-\nu)= & A_{1} \sigma_{\alpha+\beta+1}(n)+A_{2} n^{\alpha} \sigma_{-\alpha+\beta+1}(n)+A_{3} n^{\beta} \sigma_{\alpha-\beta+1}(n) \\
& +A_{4} n^{\alpha+\beta} \sigma_{-\alpha-\beta+1}(n)+O\left(n^{\omega_{1}}(\log n)^{3+\alpha}\right)
\end{aligned}
$$

where $A_{1}, A_{2}, A_{3}$ and $A_{4}$ are as defined in (61), and $\omega_{1}=3 / 4+(\alpha+\beta) / 2$ if $\alpha+\beta<1 / 2, \omega_{1}=1 / 2+\alpha+\beta$ if $\alpha+\beta \geqq 1 / 2$, and $\kappa=1$ if $\alpha+\beta=1 / 2$ and 0 otherwise.

In the case $\alpha+\beta \geqq 1 / 2$ it is possible that the error term may absorb part of the approximating function.

The author wishes to express his indebtedness to Dr. Estermann for having suggested this investigation.

Notation. $k, l, m, r, u$ are positive integers. $\tau$ is a complex number. $\theta, \vartheta$ are real numbers. $\lambda$ is a positive number less than 1 .

Wherever the $O$-notation is used, the relations are uniform with respect to all variables except $\lambda, \alpha$ and $\beta$.

$\xi_{\nu}$ stands for $e^{2 \pi i / \nu}$.

Throughout $a$ and $q$ are positive integers such that $a<q,(a, q)=1$; these restrictions on $a$ are implied automatically whenever $a$ is the variable of summation.

Preliminary results. We define, for $\mathscr{g}(\tau)>0$,

$$
f_{\lambda}(\tau)=\sum_{m=1}^{\infty} \sigma_{\lambda}(m) e^{2 \pi i m \tau}
$$

Then, provided that $\mathscr{I}(\boldsymbol{\tau})>0$,

$$
g(\tau)=f_{\alpha}(\tau) f_{\beta}(\tau)=\sum_{m=1}^{\infty} s(m) e^{2 \pi i m \tau}
$$

where

$$
s(m)=\sum_{k, l, r, u ; k l+r u=m} k^{\alpha} r^{\beta}=\sum_{\nu=1}^{m-1} \sigma_{\alpha}(\nu) \sigma_{\beta}(m-\nu) .
$$

Hence

$$
s(n)=\int g(\tau) e^{-2 \pi i n \tau} d \tau
$$

with the integration taken over the line segment $(i / n, i / n+1)$. Writing $\tau=\theta+i / n,(2)$ becomes

$$
s(n)=e^{2 \pi} \int_{0}^{1} g(\theta+i / n) e^{-2 \pi i n \theta} d \theta .
$$


We dissect $(0,1)$ into Farey arcs of order $n^{1 / 2}$, and denote the typical arc by $M_{a, q}$. It is well known that if $\theta$ is in $M_{a, q}$, we can write $\theta=a / q+\vartheta$, where

$$
-B_{1} q^{-1} n^{-1 / 2} \leqq \vartheta \leqq B_{2} q^{-1} n^{-1 / 2}
$$

with $1 / 2 \leqq B_{1} \leqq 1,1 / 2 \leqq B_{2} \leqq 1$. Then, by (3),

$$
s(n)=e^{2 \pi} \sum_{q \leqq n^{1 / 2}} \int_{-q^{-1} n^{-1 / 2}}^{q^{-1} n^{-1 / 2}}\left\{\sum_{a} \phi \xi_{q}^{-n a} g(a / q+\eta)\right\} e^{-2 \pi i n \vartheta} d \vartheta
$$

where

$$
\eta=i / n+\vartheta,
$$

and $\phi=\phi(n, a, q, \vartheta)=1$ or 0 according as $a / q+\vartheta$ does, or does not, belong to $M_{a, q}$.

We define

$$
\begin{aligned}
h_{n, q}(\eta) & =\sum_{a} \phi \xi_{q}^{-n a} g(a / q+\eta), \\
H_{n, q} & =\int_{-q^{-1} n^{-1 / 2}}^{q^{-1} n^{-1 / 2}} h_{n, q}(\eta) e^{-2 \pi i n \vartheta} d \vartheta .
\end{aligned}
$$

Then

$$
s(n)=e^{2 \pi} \sum_{q \leqq n^{1 / 2}} H_{n, q}
$$

Our first objective is to find an approximating function for $f_{\lambda}(a / q+\eta)$ and to express the resulting error term in suitable form (see (39)).

By Mellin's transformation formula

$$
f_{\lambda}(a / q+\eta)=\sum_{m=1}^{\infty} \sigma_{\lambda}(m) \xi_{q}^{a m} e^{2 \pi i m \eta}=\frac{1}{2 \pi i} \int_{2-i \infty}^{2+i \infty} \Gamma(s) z^{-s} F_{\lambda}(s) d s
$$

where

$$
z=-2 \pi i \eta
$$

and

$$
F_{\lambda}(s)=\sum_{m=1}^{\infty} \sigma_{\lambda}(m) m^{-s} \xi_{q}^{m a} .
$$

The functional equation for $F^{j}(s)$. We have that

$$
\begin{aligned}
F_{\lambda}(s) & =F_{\lambda}\left(s ; \xi_{q}^{a}\right)=\sum_{k, l} k^{\lambda} \xi_{q}^{a k l} k^{-s} l^{-s}=\sum_{b=1}^{q} \sum_{k, l ; l \neq b(\bmod q)} \xi_{q}^{a k l} k^{\lambda-s} l^{-s} \\
& =\sum_{b=1}^{q} \sum_{k=1}^{\infty} \xi_{q}^{a b k} k^{\lambda-s} \sum_{l=1 ; l=b(\bmod q)}^{\infty} l^{-s}=\sum_{b=1}^{q} \zeta\left(s-\lambda ; \xi_{q}^{a b}\right) \zeta(s ; b, q)
\end{aligned}
$$


where

$$
\zeta\left(s ; \xi_{q}^{b}\right)=\sum_{k=1}^{\infty} \xi_{q}^{b k} k^{-s}
$$

and

$$
\zeta(s ; b, q)=\sum_{k=1 ; k=1}^{\infty} k_{b(\bmod q)} k^{-s}
$$

We put $G(s)=-i(2 \pi)^{-1} \Gamma(1-s)$. Then

$$
\zeta(s ; b, q)=G(s) q^{-s}\left(e^{i \pi s / 2} \zeta\left(1-s ; \xi_{q}^{b}\right)-e^{-i \pi s / 2} \zeta\left(1-s ; \xi_{q}^{-b}\right)\right)
$$

and

$$
\zeta\left(s ; \xi_{q}^{b}\right)=G(s) q^{1-s}\left(e^{i \pi s / 2} \zeta(1-s ;-b, q)-e^{-i \pi s / 2} \zeta(1-s ; b, q)\right) .
$$

Equation (14) is proved in the same way as the functional equation for Riemann's $\zeta$-function, and (15) follows from (14). We apply (14) and (15) to (11) and obtain

$$
\begin{aligned}
F_{\lambda}(s)= & G(s) G(s-\lambda) q^{1+\lambda-2 s} \sum_{b=1}^{q}\left(e^{i \pi(o-\lambda) / 2} \zeta(1+\lambda-s ;-a b, q)\right. \\
& \left.-e^{-i \pi(s-\lambda) / 2} \zeta(1+\lambda-s ; a b, q)\right)\left(e^{i \pi s / 2} \zeta\left(1-s ; \xi_{q}^{b}\right)-e^{-i \pi s / 2} \zeta\left(1-s ; \xi_{q}^{-b}\right)\right) \\
= & G(s) G(s-\lambda) q^{1+\lambda-2 s}\left(\Sigma_{1}+\Sigma_{2}+\Sigma_{3}+\Sigma_{4}\right)
\end{aligned}
$$

where

$$
\begin{aligned}
\Sigma_{1} & =e^{i \pi(2 \sigma-\lambda) / 2} \sum_{b=1}^{q} \zeta(1+\lambda-s ;-a b, q) \zeta\left(1-s ; \xi_{q}^{b}\right) \\
\Sigma_{2} & =e^{-i \pi(2 \sigma-\lambda) / 2} \sum_{b=1}^{q} \zeta(1+\lambda-s ; a b, q) \zeta\left(1-s ; \xi_{q}^{-b}\right) \\
& =e^{-i \pi(2 s-\lambda) / 2} \sum_{b=1}^{q} \zeta(1+\lambda-s ;-a b, q) \zeta\left(1-s ; \xi_{q}^{b}\right), \\
\Sigma_{3} & =-e^{i \pi \lambda / 2} \sum_{b=1}^{q} \zeta(1+\lambda-s ; a b, q) \zeta\left(1-s ; \xi_{q}^{b}\right), \\
\Sigma_{4} & =-e^{-i \pi \lambda / 2} \sum_{b=1}^{q} \zeta(1+\lambda-s ;-a b, q) \zeta\left(1-s ; \xi_{q}^{-b}\right) \\
& =-e^{-i \pi \lambda / 2} \sum_{b=1}^{q} \zeta(1+\lambda-s ; a b, q) \zeta\left(1-s ; \xi_{q}^{b}\right) .
\end{aligned}
$$

Thus 


$$
\begin{aligned}
F_{\lambda}(s)= & 2 G(s) G(s-\lambda) q^{1+\lambda-2 s} \\
& \cdot\left\{\cos \left(s-\frac{1}{2} \lambda\right) \pi \sum_{b=1}^{q} \zeta(1+\lambda-s ;-a b, q) \zeta\left(1-s ; \xi_{q}^{b}\right)\right. \\
& \left.\quad-\cos \frac{1}{2} \lambda \pi \sum_{b=1}^{q} \zeta(1+\lambda-s ; a b, q) \zeta\left(1-s ; \xi_{q}^{b}\right)\right\} .
\end{aligned}
$$

We now define $a^{\prime}$ by

$$
a a^{\prime} \equiv 1(\bmod q),
$$$$
0<a^{\prime} \leqq q ;
$$

then if $r$ is the least positive residue $\bmod q$ of $-a b$, we have $r a^{\prime} \equiv-b a a^{\prime}$ $\equiv-b(\bmod q)$, and this together with (11) enables us to rewrite $(16)$ as

$$
\begin{aligned}
F_{\lambda}\left(s ; \xi_{q}^{a}\right)= & 2 G(s) G(s-\lambda) q^{1+\lambda-2 s}\left\{\cos \left(s-\frac{1}{2} \lambda\right) \pi \cdot F_{\lambda}\left(1+\lambda-s ; \xi_{q}^{a^{\prime}}\right)\right. \\
& \left.-\cos \frac{1}{2} \lambda \pi \cdot F_{\lambda}\left(1+\lambda-s ; \xi_{q}^{a^{\prime}}\right)\right\}
\end{aligned}
$$

the required functional equation for $F_{\lambda}(s)$.

The poles of $F_{\lambda}(s)$ at $s=1$ and $s=1+\lambda$. It is easily seen that

Further, by (11),

$$
\zeta(s ; q, q)=q^{-s} \zeta(s), \quad \sum_{b=1}^{q} \zeta\left(s ; \xi_{q}^{a b}\right)=q^{1-s} \zeta(s) .
$$

$$
F_{\lambda}(s)-q^{-s} \zeta(s) \sum_{b=1}^{q} \zeta\left(s-\lambda ; \xi_{q}^{a b}\right)=\sum_{b=1}^{q} \zeta\left(s-\lambda ; \xi_{q}^{a b}\right)\left\{\zeta(s ; b, q)-q^{-s} \zeta(s)\right\},
$$

and, by the above, since the right-hand side is a regular function of $s$ in the neighborhood of $s=1$, the residues of $F_{\lambda}(s)$ and $q^{1+\lambda-2 s} \zeta(s) \zeta(s-\lambda)$ at $s=1$ are the same, namely

$$
q^{\lambda-1} \zeta(1-\lambda)
$$

It is clear from (11) that we can also write

$$
F_{\lambda}(s)=\sum_{b=1}^{q} \zeta(s-\lambda ; b, q) \zeta\left(s ; \xi_{q}^{a \grave{b}}\right) .
$$

This, combined with the above method, enables us to prove in exactly the same way that the residue of $F_{\lambda}(s)$ at $s=1+\lambda$ is

$$
q^{-\lambda-1} \zeta(1+\lambda)
$$

Let $R_{\lambda}$ be the sum of residues of $\Gamma(s) z^{-8} F_{\lambda}(s)$ at $s=1, s=1+\lambda$. It follows from (18) and (19) that

$$
R_{\lambda}=\Gamma(1+\lambda) \zeta(1+\lambda)(q z)^{-1-\lambda}+\zeta(1-\lambda) q^{\lambda}(q z)^{-1}
$$


and from (8) that

$$
f_{\lambda}(a / q+\eta)-R_{\lambda}=F_{\lambda}(0)+\frac{1}{2 \pi i} \int_{-1 / 2-i \infty}^{-1 / 2+i \infty} \Gamma(s) z^{-s} F_{\lambda}(s) d s .
$$

We proceed to evaluate the two expressions on the right of (21).

Calculation of $F_{\lambda}(0)$. We rewrite (17) in the form

$$
\begin{aligned}
\frac{F_{\lambda}\left(s ; \xi_{q}^{a}\right)}{2 G(s) G(s-\lambda) q^{1+\lambda-2 s}}= & \left(\cos \left(s-\frac{1}{2} \lambda\right) \pi-\cos \frac{1}{2} \lambda \pi\right) F_{\lambda}\left(1-s+\lambda ; \xi_{q}^{-a^{\prime}}\right) \\
& +\cos \frac{1}{2} \lambda \pi\left[F_{\lambda}\left(1-s+\lambda ; \xi_{q}^{-a^{\prime}}\right)\right. \\
& \left.-F_{\lambda}\left(1-s+\lambda ; \xi_{q}^{a^{\prime}}\right)\right] .
\end{aligned}
$$

We observe that $G(0) G(-\lambda)=-\Gamma(1+\lambda) /(2 \pi)^{2+\lambda}$, and that, by (19),

$$
\begin{aligned}
\lim _{s \rightarrow 0}\left\{\left(\cos \left(s-\frac{1}{2} \lambda\right) \pi\right.\right. & \left.\left.-\cos \frac{1}{2} \lambda \pi\right) F_{\lambda}\left(1-s+\lambda ; \xi_{q}^{-a^{\prime}}\right)\right\} \\
& =\lim _{s \rightarrow 0}\left(s \pi \sin \frac{1}{2} \lambda \pi+\cdots\right) F_{\lambda}\left(1-s+\lambda ; \xi_{q}^{-a^{\prime}}\right) \\
& =-\pi \sin \frac{1}{2} \lambda \pi \cdot q^{-1-\lambda} \zeta(1+\lambda) .
\end{aligned}
$$

This disposes of the first term on the right of (22). As for the second, we have, by (11), that

$$
\begin{aligned}
\lim _{s \rightarrow 0}\left(F_{\lambda}\left(1-s+\lambda ; \xi_{q}^{-a^{\prime}}\right)\right. & \left.-F_{\lambda}\left(1-s+\lambda ; \xi_{q}^{a^{\prime}}\right)\right) \\
& =\lim _{s \rightarrow 1}\left(F_{\lambda}\left(s+\lambda ; \xi_{q}^{-a^{\prime}}\right)-F_{\lambda}\left(s+\lambda ; \xi_{q}^{a^{\prime}}\right)\right) \\
& =\lim _{s \rightarrow 1} \sum_{b=1}^{q} \zeta(s+\lambda ; b, q)\left(\zeta\left(s ; \xi_{q}^{-a^{\prime} b}\right)-\zeta\left(s ; \xi_{q}^{a^{\prime} b}\right)\right) \\
& =\sum_{b=1}^{q} \zeta(1+\lambda ; b, q)\left(\zeta\left(1 ; \xi_{q}^{-a^{\prime} b}\right)-\zeta\left(1 ; \xi_{q}^{a^{\prime} b}\right)\right) \\
& =\sum_{b=1}^{q-1} E_{b}\left(\zeta\left(1 ; \xi_{q}^{-a^{\prime} b}\right)-\zeta\left(1 ; \xi_{q}^{a^{\prime} b}\right)\right)
\end{aligned}
$$

where, for $0<b<q$,

$$
\begin{aligned}
E_{b} & =\zeta(1+\lambda ; b, q)=\sum_{r=0}^{\infty}(b+r q)^{-1-\lambda}<\sum_{r=0}^{\infty} b^{-1-\lambda}(r+1)^{-1-\lambda} \\
& =b^{-1-\lambda} \zeta(1+\lambda) .
\end{aligned}
$$


Now if $r_{q}(m)$ is the least non-negative residue $\bmod q$ of $m$,

$$
\begin{aligned}
\zeta\left(1 ; \xi_{q}^{-a^{\prime} b}\right)-\zeta\left(1 ; \xi_{q}^{a^{\prime} b}\right) & =\log \frac{1-\xi_{q}^{a^{\prime} b}}{1-\xi_{q}^{-a^{\prime} b}}=\log \left(-\xi_{q}^{a^{\prime} b}\right)=i\left(-\pi+\frac{2 \pi r_{q}\left(a^{\prime} b\right)}{q}\right) \\
& =2 \pi i\left(r_{q}\left(a^{\prime} b\right)-\frac{1}{2} q\right) / q
\end{aligned}
$$

so that the right-hand side of (24) can be written

$$
\frac{2 \pi i}{q} \sum_{b=1}^{q-1}\left(r_{q}\left(a^{\prime} b\right)-\frac{1}{2} q\right) E_{b}
$$

Moreover, for any integer $m$

$$
r_{q}(m)-\frac{1}{2} q=\sum_{\nu=1}^{q} c_{\nu} \xi_{q}^{v n}
$$

where

$$
c_{q}=-\frac{1}{2}, \quad c_{\nu}=\left(\xi_{q}^{-\nu}-1\right)^{-1} \quad(0<\nu<q)
$$

for

$$
\begin{array}{rlr}
c_{\nu} & =q^{-1} \sum_{l=1}^{q}\left\{r_{q}(l)-\frac{1}{2} q\right\} \xi_{q}^{-l \nu}=-\frac{1}{2}+q^{-1} \sum_{l=1}^{q-1}\left(l-\frac{1}{2} q\right) \xi_{q}^{-l \nu} \\
& =-\frac{1}{2}+q^{-1} \sum_{l=1}^{q-1} l \xi_{q}^{-l \nu}-\frac{1}{2} \sum_{l=1}^{q-1} \xi_{q}^{-l \nu} \\
& =-\frac{1}{2}+q^{-1} \frac{(q-1) q}{2}-\frac{1}{2}(q-1)=-\frac{1}{2} \quad(\nu=q), \\
& =-\frac{1}{2}+\left(\xi_{q}^{-\nu}-1\right)^{-1}+\frac{1}{2}=\left(\xi_{q}^{-\nu}-1\right)^{-1} \quad(0<\nu<q) .
\end{array}
$$

Thus the right-hand side of (24) becomes

(27)

$$
\sum_{\nu=1}^{q}\left|c_{\nu}\right| \leqq \frac{1}{2}+\sum_{1 \leqq \nu \text { 位 }} \frac{1}{\sin \pi \nu / q}<\frac{1}{2}+\frac{1}{2} q \sum_{1 \leqq \nu \leqq q / 2} \frac{1}{\nu}<q \log (2 q),
$$

so that, by (25), 
(28)

$$
\left|\sum_{1 \leqq b \leqq q-1 ; 1 \leqq \nu \leqq q} c_{\nu} E_{b}\right| \leqq\left(\sum_{\nu=1}^{q}\left|c_{\nu}\right|\right)\left(\sum_{b=1}^{q-1} E_{b}\right)<\zeta^{2}(1+\lambda) q \log (2 q) .
$$

If now we combine equations (22), (23), (24) and (27), we obtain

$$
\begin{aligned}
F_{\lambda}(0)= & -\Gamma(1+\lambda)(2 \pi)^{-2-\lambda}\left\{-2 \pi \sin \frac{1}{2} \pi \lambda \cdot \zeta(1+\lambda)\right. \\
& \left.+4 \pi i q^{\lambda} \cos \frac{1}{2} \lambda \pi \sum_{l=1}^{\lambda}\left(\sum_{1 \leqq b \leqq q-1 ; 1 \leqq \nu \leqq q ; b \nu \exists l(\bmod q)} c_{\nu} E_{b}\right) \xi_{q}^{a^{\prime} l}\right\} \\
= & \sum_{l=1}^{q} d_{l} \xi_{q}^{a^{\prime} l}
\end{aligned}
$$

where$$
d_{l}=\frac{-2 i \Gamma(1+\lambda) \cos \lambda \pi / 2}{(2 \pi)^{1+\lambda}} q^{\lambda} \sum_{1 \leqq b \leqq q-1 ; 1 \leqq \nu \leqq q ; b v=l(\bmod q)} c_{\nu} E_{b}(1 \leqq l \leqq q-1)
$$$$
\text { (30) }=\frac{\Gamma(1+\lambda) \sin \lambda \pi / 2}{(2 \pi)^{1+\lambda}}-\frac{2 i \Gamma(1+\lambda) \cos \lambda \pi / 2}{(2 \pi)^{1+\lambda}} \sum_{1 \leqq b \leqq q-1 ; 1 \leqq \nu \leqq q ; b \nu \equiv l(\bmod q)} c_{\nu} E_{b}
$$$$
(l=q) \text {, }
$$

so that, by (28),

$$
\sum_{l=1}^{q}\left|d_{l}\right|=O\left(q^{1+\lambda} \log (2 q)\right) .
$$

Calculation of the integral in (21). We denote the integral under consideration by $J_{\lambda}$. By (17), (10) and the definition of $G(s)$,

$$
\begin{aligned}
J_{\lambda}= & \int_{-1 / 2-i \infty}^{-1 / 2+i \infty} 2 G(s) G(s-\lambda) q^{1+\lambda-2 s} \Gamma(s) z^{-\cdot}\left\{\cos \left(s-\frac{1}{2} \lambda\right) \pi\right. \\
& \left.\cdot F_{\lambda}\left(1-s+\lambda ; \xi_{q}^{-a^{\prime}}\right)-\cos \frac{1}{2} \lambda \pi F_{\lambda}\left(1-s+\lambda ; \xi_{q}^{a^{\prime}}\right)\right\} d s \\
= & \sum_{m=1}^{\infty} \sigma_{\lambda}(m) \xi_{q}^{-a^{\prime} m} e_{m}-\sum_{m=1}^{\infty} \sigma_{\lambda}(m) \xi_{q}^{a^{\prime} m} \epsilon_{m},
\end{aligned}
$$

where

$$
e_{m}=-\left(\frac{q}{2 \pi m}\right)^{1+\lambda} \int_{-1 / 2-i \infty}^{-1 / 2+i \infty}\left(\frac{4 \pi^{2} m}{q^{2} z}\right)^{s} \frac{\Gamma(1+\lambda-s)}{\sin \pi s} \cos \left(s-\frac{1}{2} \lambda\right) \pi d s
$$

and

$$
\epsilon_{m}=-\left(\frac{q}{2 \pi m}\right)^{1+\lambda} \cos \frac{1}{2} \lambda \pi \int_{-1 / 2-i \infty}^{-1 / 2+i \infty}\left(\frac{4 \pi^{2} m}{q^{2} z}\right)^{s} \frac{\Gamma(1+\lambda-s)}{\sin \pi s} d s
$$


Lemia 1. If $R(\delta)>0$, then

$$
\int_{-1 / 2-i \infty}^{-1 / 2+i \infty} \delta^{s} \frac{\Gamma(1+\lambda-s)}{\sin \pi s} d s=O\left(|\delta|^{-1 / 2}\right),
$$

and

$$
\int_{-1 / 2-i \infty}^{-1 / 2+i \infty} \delta^{s} \frac{\Gamma(1+\lambda-s)}{\sin \pi s} \cos \left(s-\frac{1}{2} \lambda\right) \pi \cdot d s=O\left(|\delta|^{-1 / 2}+|\delta|^{1+\lambda} e^{-\Re \delta}\right) .
$$

Proof. Of these two results the first is trivial. To prove the second, we assume first of all that $g(\delta) \leqq 0$. By expanding $\cos (s-\lambda / 2) \pi$ it is easy to show that the second integral is equal to $\cos \frac{1}{2} \lambda \pi \int_{-1 / 2-i \infty}^{-1 / 2+i \infty} \delta^{s} \frac{\Gamma(1+\lambda-s)}{\sin \pi s} e^{i \pi s} d s-i e^{i \lambda \pi / 2} \int_{-1 / 2-i \infty}^{-1 / 2+i \infty} \delta^{s} \Gamma(1+\lambda-s) d s$.

It is clear that

$$
\int_{-1 / 2-i \infty}^{-1 / 2+i \infty} \delta^{s} \frac{\Gamma(1+\lambda-s)}{\sin \pi s} e^{i \pi s} d s=O\left(|\delta|^{-1 / 2}\right)
$$

and

$$
\int_{-1 / 2-i \infty}^{-1 / 2+i \infty} \delta^{s} \Gamma(1+\lambda-s) d s=\int_{3 / 2+\lambda-i \infty}^{3 / 2+\lambda+i \infty} \Gamma(w) \delta^{1+\lambda-w} d w=2 \pi i \delta^{1+\lambda} e^{-\lambda} .
$$

Hence the result if $\mathscr{g}(\delta) \leqq 0$; the proof for $\mathscr{g}(\delta)>0$ is similar.

We apply Lemma 1 with $\delta=4 \pi^{2} m /\left(q^{2} z\right)$ to $e_{m}$ and $\epsilon_{m}$. We note that by (4), (9) and because $|\vartheta| \leqq q^{-1} n^{-1 / 2}, q \leqq n^{1 / 2}$,

$$
R(\delta)=R\left(\frac{4 \pi^{2} m}{q^{2} z}\right)=\frac{8 \pi^{3} m}{q^{2}|z|^{2} n}=\frac{2 \pi m}{q^{2} n\left(\vartheta^{2}+n^{-2}\right)} \geqq \pi m>0,
$$

whence

$$
e^{-\Re \delta}=\exp \left(-\Re \frac{4 \pi^{2} m}{q^{2} z}\right) \leqq 2\left(\Re \frac{4 \pi^{2} m}{q^{2} z}\right)^{-2}<\frac{2}{\pi m}\left(\Re \frac{4 \pi^{2} m}{q^{2} z}\right)^{-1}=\frac{q^{2}|z|^{2} n}{4 \pi^{4} m^{2}} .
$$

It follows from Lemma 1 together with these two inequalities, and equations (33) and (34), that

$$
\left|e_{m}\right|+\left|\epsilon_{m}\right|=O\left(q^{2+\lambda}|z|^{1 / 2} m^{-(3 / 2+\lambda)}+n q^{1-\lambda}|z|^{1-\lambda} m^{-2}\right)=O\left(n(q|z|)^{1-\lambda} m^{-t}\right)
$$

where $t=\min (2,3 / 2+\lambda)$. Hence

$$
\sum_{m=1}^{\infty} \sigma_{\lambda}(m)\left\{\left|e_{m}\right|+\left|\epsilon_{m}\right|\right\}=O\left(n(q|z|)^{1-\lambda} \sum_{m=1}^{\infty} \sigma_{\lambda}(m) m^{-t}\right)=O\left(n(q|z|)^{1-\lambda}\right) .
$$

If now we put 


$$
d_{l}^{*}=\sum_{m=1 ; m \equiv-l(\bmod q)}^{\infty} \sigma_{\lambda}(m) e_{m}-\sum_{m=1 ; m \equiv l(\bmod q)}^{\infty} \sigma_{\lambda}(m) \epsilon_{m},
$$

it follows from (32) that

$$
J_{\lambda}=\sum_{l=1}^{q} d_{l}^{*} \xi_{q}^{l a^{\prime}}
$$

where, by (35) and (36),

$$
\sum_{l=1}^{q}\left|d_{l}^{*}\right|=O\left(n(q|z|)^{1-\lambda}\right) .
$$

In view of equations (29) and (37) we are now able to rewrite (21) as

$$
f_{\lambda}(a / q+\eta)-R_{\lambda}=\sum_{l=1}^{q} j_{l} \xi_{q}^{l a^{\prime}}
$$

where

$$
j_{l}=d_{l}+\frac{1}{2 \pi i} d_{l}^{*}
$$

so that, by (31) and (38),

$$
\sum_{l=1}^{q}\left|j_{l}\right|=O\left(q^{1+\lambda} \log (2 q)+n(q|z|)^{1-\lambda}\right)=O\left(n(q|z|)^{1-\lambda} \log (2 q)\right) .
$$

Calculation of $g(a / q+\eta)-R_{\alpha} R_{\beta}$. We note that $g-R_{\alpha} R_{\beta}=f_{\alpha} f_{\beta}-R_{\alpha} R_{\beta}$ $=\left(f_{\alpha}-R_{\alpha}\right) R_{\beta}+\left(f_{\beta}-R_{\beta}\right) f_{\alpha}$; hence, by (39) and (20),

$$
g(a \mid q+\eta)-R_{\alpha} R_{\beta}=\sum_{l=1}^{q} k_{l} \xi_{q}^{\alpha^{\prime} l}
$$

where $k_{1}, k_{2}, \cdots, k_{q}$ are numbers, independent of $a$, such that, by (20) and $(41)$,

$$
\begin{aligned}
\sum_{l=1}^{q}\left|k_{l}\right| & =O\left(n^{2}(q|z|)^{2-\alpha-\beta} \log ^{2}(2 q)+n(q|z|)^{-\alpha-\beta} \log (2 q)\right) \\
& =O\left(n^{1+\alpha+\beta} q^{-(\alpha+\beta)} \log ^{2} n\right) .
\end{aligned}
$$

The error term. We put

$$
T=R_{\alpha} R_{\beta} \sum_{a} \phi \xi_{q}^{-n a}
$$

and

$$
V=R_{\alpha} R_{\beta} \sum_{a} \xi_{q}^{-n a}=c_{q}(n) R_{\alpha} R_{\beta}
$$


where $c_{q}(n)$ is Ramanujan's function. We define

$$
t_{n, q}=\int_{-q^{-1} n^{-1 / 2}}^{q^{-1} n^{-1 / 2}} T e^{-2 \pi i n \vartheta} d \vartheta
$$

and

$$
v_{n, \eta}=\int_{-\infty}^{\infty} V e^{-2 \pi i n} d \vartheta
$$

We have by (5), (42) and (44) that

$$
\begin{aligned}
h_{n, q}(\eta)-T & =\sum_{a} \phi\left\{g(a \mid q+\eta)-R_{\alpha} R_{B}\right\} \xi_{q}^{-n a}=\sum_{a} \phi \sum_{l=1}^{q} k_{l} \xi_{q}^{l a^{\prime}-n a} \\
& =\sum_{l=1}^{q} k_{l} \sum_{a} \phi \xi_{q}^{l a^{\prime}-n a}
\end{aligned}
$$

It has been shown by Estermann $\left(^{5}\right)$ that an estimate for the inner sum on the right can be derived from an estimate of Kloosterman's sum. Moreover, a "best possible" estimate of $O\left(q^{1 / 2}\right)$ for Kloosterman's sum was obtained by A. Weil $\left({ }^{6}\right)$ some years ago. If then we combine Estermann's method with Weil's result we find that

$$
\sum_{a} \phi \xi_{q}^{l a^{\prime}-n a}=O\left(q^{1 / 2} \log (4 q)\right)
$$

and

$$
\sum_{a}(\phi-1) \xi_{q}^{-n a}=O\left(q^{1 / 2} \log (4 q)\right) .
$$

It follows from (43), (48) and (49) that

$$
h_{n, q}(\eta)-T=O\left(n^{1+\alpha+\beta} q^{1 / 2-\alpha-\beta} \log ^{3} n\right),
$$

and from (20), (44), (45) and (50) that

$$
T-V=R_{\alpha} R_{\beta} \sum_{a}(\phi-1) \xi_{q}^{-n a}=O\left((q|z|)^{-2-\alpha-\beta} q^{1 / 2} \log n\right) .
$$

Now it is clear from our definition of $\phi$ that $T=V$ for $|\vartheta| \leqq q^{-1} n^{-1 / 2} / 2$ so that $T-V$ can be nonzero only for $|\vartheta|>q^{-1} n^{-1 / 2} / 2$. Since, by (4) and (9), $|z|>2 \pi|\vartheta|$, we have that

$$
T-V=O\left((q|\vartheta|)^{-2-\alpha-\beta} q^{1 / 2} \log n\right),
$$

$$
\left(|\vartheta|>\frac{1}{2} q^{-1} n^{-1 / 2}\right)
$$

(5) See Estermann [3].

(6) See Weil [4]. The so-called Kloosterman sum is $\sum_{a} \xi_{q}^{l a+m a d}$. 
By (6), (46) and (51)

$$
H_{n, q}-t_{n, q}=O\left(n^{1 / 2+\alpha+\beta} q^{-1 / 2-\alpha-\beta} \log ^{3} n\right) .
$$

Furthermore, our definition of $\phi(n, a, q, \vartheta)$ implies that

$$
t_{n, q}=\int_{-\infty}^{\infty} T e^{-2 \pi i n \vartheta} d \vartheta \text {. }
$$

Hence, by (47) and (52),

$$
\begin{aligned}
l_{n, q}-v_{n, q} & =O\left(\int_{q^{-1} n^{-1 / 2} / 2}^{\infty}(q \vartheta)^{-2-\alpha-\beta} q^{1 / 2} \log n \cdot d \vartheta\right) \\
& =O\left(n^{(1+\alpha+\beta) / 2} q^{-1 / 2} \log n\right)=O\left(n^{1 / 2+\alpha+\beta} q^{-(\alpha+\beta+1 / 2)} \log n\right) .
\end{aligned}
$$

It follows from (7), (53) and (54) that

$$
\begin{aligned}
s(n)-e^{2 \pi} \sum_{q \leqq n^{1 / 2}} v_{n, q} & =O\left(n^{1 / 2+\alpha+\beta} \log ^{3} n \sum_{q \leqq n^{1 / 2}} q^{-1 / 2-\alpha-\beta}\right) \\
& =O\left(n^{\omega_{1}}(\log n)^{3+\kappa}\right)
\end{aligned}
$$

where $\omega_{1}=1 / 2+\alpha+\beta$ if $\alpha+\beta \geqq 1 / 2, \omega_{1}=1 / 4+(1+\alpha+\beta) / 2$ if $\alpha+\beta \leqq 1 / 2$, and $\kappa=1$ if $\alpha+\beta=1 / 2$ and 0 otherwise.

Finally, it is well known that

$$
c_{q}(n)=\sum_{d \mid(q, n)} \mu\left(\frac{q}{d}\right) d,
$$

so that by (47), (45), (20), (9) and (4)

$$
\begin{aligned}
\sum_{q>n^{1 / 2}} v_{n, q} & =\sum_{q>n^{1 / 2}} c_{q}(n) \int_{-\infty}^{\infty} R_{\alpha} R_{\beta} e^{-2 \pi i n \vartheta} d \vartheta=O\left(n^{1+\alpha+\beta} \sum_{q>n^{1 / 2}}\left|c_{q}(n)\right| q^{-2-\alpha-\beta}\right) \\
& =O\left(n^{1+\alpha+\beta} \sum_{q>n^{1 / 2}} q^{-2-\alpha-\beta} \sum_{d \mid(q, n)} d\right) \\
& =O\left(n^{1+\alpha+\beta} \sum_{d \mid n} d \sum_{q>n^{1 / 2}, d \mid q} q^{-2-\alpha-\beta}\right) \\
& =O\left(n^{1+\alpha+\beta} \sum_{d \mid n} d^{-1-\alpha-\beta} \sum_{m>n^{1 / 2} / d} m^{-2-\alpha-\beta}\right) \\
& =O\left(n^{(1+\alpha+\beta) / 2} \sum_{d \mid n} 1\right)=O\left(n^{\omega_{1}}\right) .
\end{aligned}
$$

Hence, by (55), 


$$
s(n)-e^{2 \pi} \sum_{q=1}^{\infty} v_{n, q}=O\left(n^{\omega_{1}}(\log n)^{3+\kappa}\right) .
$$

The dominant term. By (20)

$$
R_{\alpha} R_{\beta}=a_{1}(q z)^{-2-\alpha-\beta}+a_{2} q^{\alpha}(q z)^{-2-\beta}+a_{3} q^{\beta}(q z)^{-2-\alpha}+a_{4} q^{\alpha+\beta}(q z)^{-2}
$$

where

$$
\begin{array}{ll}
a_{1}=\Gamma(1+\alpha) \Gamma(1+\beta) \zeta(1+\alpha) \zeta(1+\beta), & a_{2}=\Gamma(1+\beta) \zeta(1+\beta) \zeta(1-\alpha) \\
a_{3}=\Gamma(1+\alpha) \zeta(1+\alpha) \zeta(1-\beta), & a_{4}=\zeta(1-\alpha) \zeta(1-\beta) .
\end{array}
$$

Lemma 2. If $z$ is as defined by (9) and (4), and if $k>1$, then

$$
\int_{-\infty}^{\infty} z^{-k} e^{-2 \pi i n \vartheta} d \vartheta=e^{-2 \pi} n^{k-1} / \Gamma(k)
$$

Proof. The integral on the left is equal to

$$
\begin{aligned}
\int_{-\infty}^{\infty}\left(\frac{2 \pi}{n}-2 \pi i \vartheta\right)^{-k} e^{-2 \pi i n \vartheta} d \vartheta & =n^{k} \int_{-\infty}^{\infty}(2 \pi-2 \pi i n \vartheta)^{-k} e^{-2 \pi} e^{2 \pi-2 \pi i n \vartheta} d \vartheta \\
& =\frac{e^{-2 \pi} n^{k-1}}{2 \pi i} \int_{2 \pi-i \infty}^{2 \pi+i \infty} e^{w} w^{-k} d w=\frac{e^{-2 \pi} n^{k-1}}{\Gamma(k)} .
\end{aligned}
$$

In view of (45), (47) and (57) $v_{n, q}$ involves four integrals of the type considered in Lemma 2, and an application of the lemma gives

$$
\begin{aligned}
e^{2 \pi} v_{n, q}=c_{q}(n)\left\{\frac{a_{1}}{\Gamma(2+\alpha+\beta)} \frac{n^{1+\alpha+\beta}}{q^{2+\alpha+\beta}}\right. & +\frac{a_{2}}{\Gamma(2+\beta)}-\frac{n^{1+\beta}}{q^{2+\beta-\alpha}} \\
& \left.+\frac{a_{3}}{\Gamma(2+\alpha)} \frac{n^{1+\alpha}}{q^{2+\alpha-\beta}}+a_{4} \frac{n}{q^{2-\alpha-\beta}}\right\} .
\end{aligned}
$$

LEMMA 3.

$$
\sum_{q=1}^{\infty} c_{q}(n) q^{-k}=\frac{1}{\zeta(k)} \sigma_{1-k}(n),
$$

Proof. The sum on the left is equal to

$$
\begin{aligned}
\sum_{q=1}^{\infty} \sum_{d|n, d| q} \mu\left(\frac{q}{d}\right) d q^{-k} & =\sum_{d \mid n} d \sum_{q=1 ; d \mid q}^{\infty} \mu\left(\frac{q}{d}\right) q^{-k}=\sum_{d \mid n} d^{1-k} \sum_{m=1}^{\infty} \mu(m) m^{-k} \\
& =\frac{\sigma_{1-k}(n)}{\zeta(k)} .
\end{aligned}
$$

It follows from (56), (59) and I.emma 3 that 


$$
\begin{aligned}
s(n)= & A_{1} n^{1+\alpha+\beta} \sigma_{-1-\alpha-\beta}(n)+A_{2} n^{1+\beta} \sigma_{-1-\beta+\alpha}(n)+A_{3} n^{1+\alpha} \sigma_{-1-\alpha+\beta}(n) \\
& +A_{4} n \sigma_{-1+\alpha+\beta}(n)+O\left(n^{\omega_{1}}(\log n)^{3+\kappa}\right),
\end{aligned}
$$

where, by (58), (59) and Lemma 3 ,

$$
\begin{aligned}
A_{1} & =\frac{\Gamma(1+\alpha) \Gamma(1+\beta)}{\Gamma(2+\alpha+\beta)} \frac{\zeta(1+\alpha) \zeta(1+\beta)}{\zeta(2+\alpha+\beta)}, & A_{2} & =\frac{1}{1+\beta} \frac{\zeta(1+\beta) \zeta(1-\alpha)}{\zeta(2+\beta-\alpha)}, \\
A_{3} & =\frac{1}{1+\alpha} \frac{\zeta(1+\alpha) \zeta(1-\beta)}{\zeta(2+\alpha-\beta)}, & A_{4} & =\frac{\zeta(1-\alpha) \zeta(1-\beta)}{\zeta(2-\alpha-\beta)} .
\end{aligned}
$$

Since $n^{k} \sigma_{-k}(n)=\sigma_{k}(n)$, (60) implies our theorem.

\section{REFERENCES}

1. H. Halberstam, Four asymptotic formulae in the theory of numbers, J. London Math. Soc. vol. 24 (1949) pp. 13-21.

2. 'T. Estermann, On the representations of a number as a sum of two products, Proc. London Math. Soc. 2 vol. 31 (1929) pp. 123-133.

3. - Vereinfachter Beweis eines Satzes von Kloosterman, Abh. Math. Sem. Hansischen Univ. vol. 7 (1929) pp. 83-98. 207.

4. A. Weil, On some exponential sums, Proc. Nat. Acad. Sci. U.S.A. vol. 34 (1948) pp. 204-

EXETER UNIVERSITY,

ENGLAND

Brown UNIVERSITY,

Providence, R. I. 